\title{
APPLICATIONS OF OPTIMALLY LOCAL INTERPOLATION TO INTERPOLATORY APPROXIMANTS AND COMPACTLY SUPPORTED WAVELETS
}

\author{
CHARLES K. CHUI AND JOHAN M. DE VILLIERS
}

\begin{abstract}
The objective of this paper is to introduce a general scheme for the construction of interpolatory approximation formulas and compactly supported wavelets by using spline functions with arbitrary (nonuniform) knots. Both construction procedures are based on certain "optimally local" interpolatory fundamental spline functions which are not required to possess any approximation property.
\end{abstract}

\section{INTRODUCTION}

Let $n \geq 3$ be any positive integer. This paper is concerned with (polynomial) spline functions of order $n$ and with knot sequence

$$
\left\{\begin{array}{l}
\mathrm{t}: \cdots \leq t_{-1} \leq t_{0} \leq \cdots, \\
\text { where } t_{i+2}-t_{i}>0, \quad i \in \mathbb{Z} .
\end{array}\right.
$$

The space of such spline functions will be denoted by $\mathcal{S}_{n, \mathbf{t}}$; and in view of the separation condition in (1.1), every function in $\mathcal{S}_{n, \mathbf{t}}$ is necessarily in $C^{n-3}(\mathbb{R})$. Hence, by setting

$$
\left\{\begin{array}{l}
x_{i}:=t_{2 i}, \quad i \in \mathbb{Z}, \\
\text { so that } \quad \mathbf{x}: \cdots<x_{-1}<x_{0}<\cdots,
\end{array}\right.
$$

it is meaningful to study the problem of interpolation from $\mathcal{S}_{n, \mathbf{t}}$ with sample points at $\mathbf{x}$.

We first consider for any integer $\nu$, with $1 \leq \nu \leq \max \{1, n-3\}$, the seemingly useless "optimally local" (i.e., minimally supported) fundamental functions $\ell_{n, \nu, i}=$ $\ell_{n, \nu, \mathbf{t}, \mathbf{x}, i}$ in $\mathcal{S}_{n, \mathbf{t}}$, that are defined (uniquely) by the interpolation conditions

$$
\ell_{n, \nu, i}\left(x_{j}\right)=\delta_{i, j}, \quad i, j \in \mathbb{Z},
$$

and the condition that $x_{i-\nu}$ is closest, from the left, to the left-hand endpoint of the interval supp $\ell_{n, \nu, i}$. For the shape of $\ell_{n, \nu, i}$ to be most "symmetric", $\nu$ must be so chosen that the sample point $x_{i}$ is closest to the middle one among the knots

Received by the editor December 17, 1993.

1991 Mathematics Subject Classification. Primary 41A05, 41A15.

Research of the first author was supported by NSF Grant DMS 92-06928 and ARO Contract DAAH 03-93-G-0047.

(C)1996 American Mathematical Society 
$\mathbf{t} \cap \operatorname{supp} \ell_{n, \nu, i}$. Hence, again by leaning toward the left if necessary, the optimal choice of $\nu$ for this purpose is

$$
i_{n}:=\max \left\{1,\left\lfloor\frac{n-2}{2}\right\rfloor\right\}
$$

(where $\lfloor x\rfloor$ denotes the largest integer not exceeding $x$ ), and we will use the notation

$$
\ell_{n, i}:=\ell_{n, i_{n}, i} .
$$

Hence, by following the notation

$$
N_{n, i}(x)=N_{n, \mathbf{t}, i}(x):=\left(t_{i+n}-t_{i}\right)\left[t_{i}, \ldots, t_{i+n}\right](\cdot-x)_{+}^{n-1}
$$

for the $n$ th-order (normalized) $B$-splines in $\mathcal{S}_{n, t}$ in [1, p. 108], we may immediately conclude that

$$
\ell_{3,1, i}(x)=\frac{N_{3,2 i-1}(x)}{N_{3,2 i-1}\left(x_{i}\right)},
$$

whereas, for $n \geq 4$, it is not difficult to see that $\ell_{n, \nu, i}$ is a linear combination of the $B$-splines

$$
N_{n, 2 i-2 \nu}, \ldots, N_{n, 2 i-2 \nu+n-4},
$$

so that

$$
\operatorname{supp} \ell_{n, \nu, i}= \begin{cases}{\left[t_{2 i-1}, x_{i+1}\right]} & \text { for } \quad n=3, \\ {\left[x_{i-\nu}, x_{i-\nu+n-2}\right]} & \text { for } \quad n \geq 4 .\end{cases}
$$

In particular, for $\nu=i_{n}$, we have

$$
\operatorname{supp} \ell_{n, i}=\left[x_{i-i_{n}}, x_{i-i_{n}+n-2}\right]= \begin{cases}{\left[x_{i-i_{n}}, x_{i+i_{n}}\right]} & \text { for even } n \geq 4, \\ {\left[x_{i-i_{n}}, x_{i+i_{n}+1}\right]} & \text { for odd } n \geq 5 .\end{cases}
$$

For $n \geq 4$, to formulate $\ell_{n, \nu, i}$ explicitly, we rely on the totally positive (and hence, nonsingular, because of the location of the sample points) $(n-3) \times(n-3)$ matrix

$$
A_{n, \nu, i}:=\left[N_{n, 2 i-2 \nu+k-1}\left(x_{i-\nu+j}\right)\right]_{1 \leq j, k \leq n-3}=\left[\begin{array}{c}
N_{n, 2 i-2 \nu}, \ldots, N_{n, 2 i-2 \nu+n-4} \\
x_{i-\nu+1}, \ldots, x_{i-\nu+n-3}
\end{array}\right]
$$

(see [18] for the notation) and the corresponding matrix

$$
\tilde{A}_{n, \nu, i}(x):=\left[\begin{array}{cc}
N_{n, 2 i-2 \nu}, & \ldots, \\
x_{i-\nu+1}, \ldots, x_{i-1}, x, x_{i+1}, \ldots, x_{i-\nu+n-3}
\end{array}\right],
$$

which is obtained by replacing the $\nu$ th row of $A_{n, \nu, i}$ with the row vector

$$
\left[N_{n, 2 i-2 \nu}(x), \ldots, N_{n, 2 i-2 \nu+n-4}(x)\right] .
$$

It is now clear that the optimally local fundamental functions $\ell_{n, \nu, i}$ are given by

$$
\ell_{n, \nu, i}(x)=\frac{\operatorname{det} \tilde{A}_{n, \nu, i}(x)}{\operatorname{det} A_{n, \nu, i}}, \quad n \geq 4, \quad i \in \mathbb{Z}, \quad 1 \leq \nu \leq n-3 .
$$

Graphs of $\ell_{n, \nu, i}$ will be displayed in $\S 4$. 
These basic functions give rise to the "optimally local" interpolation operator $L_{n, \nu}$, defined on the space $C(\mathbb{R})$ of continuous functions by

$$
\left(L_{n, \nu} f\right)(x):=\sum_{i \in \mathbb{Z}} f\left(x_{i}\right) \ell_{n, \nu, i}(x), \quad f \in C(\mathbb{R}) .
$$

From the interpolatory property (1.3), we have

$$
\left(L_{n, \nu} f-f\right)\left(x_{j}\right)=0, \quad j \in \mathbb{Z} .
$$

It is important to remark, however, that the operator $L_{n, \nu}$ does not provide a good approximation. In fact, it does not even reproduce constants. In this paper, we will give two somewhat unusual applications of $L_{n, \nu}$ and particularly, in $\S 3$, of

$$
L_{n}:=L_{n, i_{n}} .
$$

The first is to give a simpler algebraic procedure than that of Dahmen, Goodman, and Micchelli $[11,12]$ for constructing locally supported interpolants from $S_{n, \mathbf{t}}$ with simple knot sequence t, i.e.,

$$
t_{i+1}>t_{i}, \quad i \in \mathbb{Z}
$$

that possess approximation order $\rho$, for any desirable $\rho \leq n$; and the second is to obtain explicit expressions of compactly supported spline wavelets with arbitrary nonuniform knots which are allowed to coalesce up to the separation condition in (1.1). This class of wavelets includes the minimally supported spline wavelets for uniform knots introduced in [9] and the extension to nonuniform simple knots by Buhmann and Micchelli [2] (where only existence was established). It does not, however, give immediately the spline wavelets on bounded intervals introduced in [7], and a somewhat more general consideration by Lyche and Mørken [21].

The idea behind our constructive scheme for compactly supported interpolants with any desirable order of approximation is originated from [6]. However, since we are interested in smallest possible and specific supports as designated by the index $\nu$, great care has to be taken in the construction of the quasi-interpolants that give a tight match with the optimally local interpolation operator $L_{n, \nu}$. The techniques we will introduce are based on those in [14,15], where $x_{i}=t_{(n-1) i}, n \geq 3$, in (1.2) was considered instead. (For further development of this approach, the reader is referred to $[16,17]$.) We also remark that the resulting interpolatory approximants so constructed in this paper are more attractive than those constructed in [4] by applying the quasi-interpolants in [3] directly. By "blending" with the operators $L_{n, \nu}$, the constructive scheme to be developed in $\S 2$ becomes quite easy to use. For instance, to construct the optimally local interpolatory approximants of Dahmen, Goodman, and Micchelli [11] for the cases $3 \leq n \leq 5$, we reduce the dimension of the system of linear equations to be solved by twice the value of the approximation order.

For the construction of spline wavelets, it was already noted in [8] and formally established in [10] that the wavelet space of order $m$ with knots $\frac{1}{2} \mathbb{Z}$ is isomorphic to the subspace of cardinal splines of order $n=2 m$ (with the same knots) that 
vanish at $\mathbb{Z}$ (see $[5$, Theorem 6.3]). The formulation of this result in the setting of nonuniform simple knots is instrumental to the existence proof in [2]. We will again make use of this isomorphism framework; but with the aid of the optimally local interpolation operator $L_{n}$, for $n=2 m$, it is quite simple to construct compactly supported spline functions in $\mathcal{S}_{n, \mathbf{t}}$ that vanish at the sample sequence $\mathbf{x}$. Only the selection of the sample spline function that gives rise to the corresponding wavelets with minimum supports requires some tricky manipulation. This consideration will be discussed in $\S 3$.

In this paper, we only consider interpolation of function values. If some appropriate multiplicities of order at least 1 are assigned to the knots $t_{i}$ individually, it is also easy to formulate the corresponding optimally local Hermite interpolants with Taylor data of possibly different lengths at different sample points $x_{i}$. This flexibility allows the possibility of constructing locally supported Hermite interpolatory approximants as well as Hermite-wavelets that generalize those of Goodman, Lee, and Tang [19] to the setting of nonuniform knots. A thorough treatment of this general framework requires more careful investigation.

\section{LOCAL INTERPOLATORY SPLINE INTERPOLANTS}

In this section, we demonstrate how the blending formula introduced in [6] can be used to construct a local interpolatory spline approximant, with arbitrary order $(\leq n)$ of exactness on polynomial reproduction, by matching an explicitly constructed optimally local spline approximant with the optimally local spline interpolant discussed in Section 1. In addition, it is shown that the resulting approximation operator is, for $3 \leq n \leq 5$, the optimally local operator for which existence and uniqueness was established by Dahmen, Goodman, and Micchelli [11].

2.1. An optimally local approximation operator. Our first objective is to explicitly construct, for a knot sequence $\mathbf{t}$ satisfying (1.15) and for integers $n, \rho, \lambda$, satisfying $n \geq 3,1 \leq \rho \leq n, \lambda \in \mathbb{Z}$, a sequence of spline functions

$$
u_{\rho, \lambda, i}=u_{\rho, \lambda, n, \mathbf{t}, i} \in \mathcal{S}_{n, \mathbf{t}}, \quad i \in \mathbb{Z},
$$

which possess the following two properties:

$$
\text { supp } u_{\rho, \lambda, i} \subseteq\left[t_{2 i-\lambda}, t_{2 i-\lambda+n+2 \rho-1}\right]
$$

and the corresponding approximation operator $U_{\rho, \lambda}=U_{\rho, \lambda, n, \mathbf{t}}$, defined by

$$
\left(U_{\rho, \lambda} f\right)(x):=\sum_{i} f\left(x_{i}\right) u_{\rho, \lambda, i}(x)
$$

reproduces all polynomials of order $\rho$ (or degree $\leq \rho-1$ ), namely,

$$
U_{\rho, \lambda} p=p, \quad p \in \pi_{\rho-1} .
$$

Hence, we write $u_{\rho, \lambda, i}$ as a $B$-spline series

$$
u_{\rho, \lambda, i}=\sum_{j=-\lambda}^{-\lambda+2 \rho-1} a_{i, j} N_{n, 2 i+j},
$$


where the $B$-spline coefficients $a_{i, j}=a_{\rho, \lambda, n, \mathbf{t}, i, j}$ will now be shown to be uniquely determined by the polynomial reproduction condition (2.3).

Recalling the standard result (see e.g. [22, p. 125]) that

$$
x^{k}=\frac{(-1)^{k} k !}{(n-1) !} \sum_{i} \psi_{n, i}^{(n-1-k)}(0) N_{n, i}(x) \quad x \in \mathbb{R}, \quad k=0,1, \ldots, n-1,
$$

with $\psi_{i}=\psi_{n, \mathbf{t}, i} \in \pi_{n-1}$ defined by

$$
\psi_{i}(x):=\prod_{q=1}^{n-1}\left(x-t_{i+q}\right),
$$

we find, after some manipulation, that the $B$-spline coefficients in (2.4) are uniquely determined by solving, for fixed $i \in \mathbb{Z}$ and $r \in\{0,1\}$, the $\rho \times \rho$ (transposed) Vandermonde linear system

$$
\sum_{j=-\left\lfloor\frac{\lambda+r}{2}\right\rfloor}^{-\left\lfloor\frac{\lambda+r}{2}\right\rfloor+\rho-1}\left(x_{i-j}\right)^{k} a_{i-j, 2 j+r}=\frac{(-1)^{k} k !}{(n-1) !} \psi_{2 i+r}^{(n-1-k)}(0), \quad k=0,1, \ldots, \rho-1 .
$$

As noted also in [15, pp. 114-115], a Vandermonde-type coefficient matrix similar to the one implied by (2.7) can be explicitly inverted; indeed, introducing the basic polynomials $\phi_{i, r, j}=\phi_{\rho, \lambda, \mathbf{x}, i, r, j} \in \pi_{\rho-1}$ by

$$
\phi_{i, r, j}(x):=\prod_{j \neq q=-\left\lfloor\frac{\lambda+r}{2}\right\rfloor}^{-\left\lfloor\frac{\lambda+r}{2}\right\rfloor+\rho-1}\left(x-x_{i-q}\right),
$$

we can now show that the resulting $B$-spline series for $u_{\rho, \lambda, i}$ has the form

$$
u_{\rho, \lambda, i}=\sum_{r=0}^{1} \sum_{j=-\left\lfloor\frac{\lambda+r}{2}\right\rfloor}^{-\left\lfloor\frac{\lambda+r}{2}\right\rfloor+\rho-1} a_{i, 2 j+r} N_{n, 2 i+2 j+r},
$$

where

$$
a_{i, 2 j+r}=\frac{1}{(n-1) !} \frac{\sum_{k=0}^{\rho-1}(-1)^{k} \phi_{i+j, r, j}^{(k)}(0) \psi_{2 i+2 j+r}^{(n-1-k)}(0)}{\phi_{i+j, r, j}\left(x_{i}\right)} .
$$

Next, we proceed to show that the formula (2.10) can be expressed explicitly in terms of the knot distributions. We shall need the polynomial identity in Lemma 2.1 below which is an extension of Lemma 3.3 in [14], in which two polynomials of the same degree were considered. To prove Lemma 2.1, we shall employ the known result (see e.g. [14, Lemma 3.2]) that, with $\kappa$ denoting a positive integer and $\left\{\gamma_{1}, \ldots, \gamma_{\kappa}\right\} \subset \mathbb{R}$, we have

$$
\prod_{r=1}^{\kappa}\left(x-\gamma_{r}\right)=\sum_{k=0}^{\kappa}(-1)^{k} \sigma_{k}\left(\gamma_{1}, \ldots, \gamma_{\kappa}\right) x^{\kappa-k}
$$

where $\sigma_{k}\left(\gamma_{1}, \ldots, \gamma_{\kappa}\right)$ denotes the classical symmetric function defined by

$\sigma_{k}\left(\gamma_{1}, \ldots, \gamma_{\kappa}\right):= \begin{cases}\sum_{i_{1}=1}^{\kappa-k+1} \gamma_{i_{1}} \sum_{i_{2}=i_{1}+1}^{\kappa-k+2} \gamma_{i_{2}} \cdots \sum_{i_{k}=i_{k-1}+1}^{\kappa} \gamma_{i_{k}} & \text { for } k=1, \ldots, \kappa, \\ 1 & \text { for } k=0 .\end{cases}$ 
Lemma 2.1. Let $\mu$ and $\nu$ denote positive integers with $1 \leq \mu \leq \nu$, and suppose that $\left\{\alpha_{1}, \ldots, \alpha_{\mu}\right\},\left\{\beta_{1}, \ldots, \beta_{\nu}\right\} \subset \mathbb{R}$. Then the polynomials $p \in \pi_{\mu}$ and $q \in \pi_{\nu}$ defined by

$$
p(x):=\prod_{r=1}^{\mu}\left(x-\alpha_{r}\right), \quad q(x):=\prod_{r=1}^{\nu}\left(x-\beta_{r}\right),
$$

satisfy the identity

$$
\sum_{k=0}^{\mu}(-1)^{k} p^{(k)}(0) q^{(\nu-k)}(0)=(\nu-\mu) ! \sum_{i_{1}=1}^{\nu}\left(\beta_{i_{1}}-\alpha_{1}\right) \sum_{\substack{i_{2}=1 \\ i_{2} \neq i_{1}}}^{\nu}\left(\beta_{i_{2}}-\alpha_{2}\right) \ldots \sum_{\substack{i_{\mu}=1 \\ i_{\mu} \neq i_{1}, \cdots, i_{\mu-1}}}^{\nu}\left(\beta_{i_{\mu}}-\alpha_{\mu}\right) .
$$

Proof. Applying the identity (2.11) to the polynomials in (2.13), we find

$$
\begin{aligned}
& \sum_{k=0}^{\mu}(-1)^{k} p^{(k)}(0) q^{(\nu-k)}(0) \\
& \quad=(-1)^{\mu} \sum_{k=0}^{\mu}(-1)^{k} k !(\nu-k) ! \sigma_{\mu-k}\left(\alpha_{1}, \ldots, \alpha_{\mu}\right) \sigma_{k}\left(\beta_{1}, \ldots, \beta_{\nu}\right) .
\end{aligned}
$$

Hence, by adopting the vector notation $\underline{\alpha}=\left(\alpha_{1}, \ldots, \alpha_{\mu}\right)$, and defining the function $F: \mathbb{R}^{\mu} \rightarrow \mathbb{R}$ by

$$
F(\underline{\alpha}):=\sum_{i_{1}=1}^{\nu}\left(\beta_{i_{1}}-\alpha_{1}\right) \sum_{\substack{i_{1}=1 \\ i_{2} \neq i_{1}}}^{\nu}\left(\beta_{i_{2}}-\alpha_{2}\right) \cdots \sum_{\substack{i_{\mu}=1 \\ i_{\mu} \neq i_{1}, \ldots, i_{\mu-1}}}^{\nu}\left(\beta_{i_{\mu}}-\alpha_{\mu}\right),
$$

it will clearly suffice to show that

$$
F(\underline{\alpha})=\frac{\nu !}{(\nu-\mu) !} \sum_{k=0}^{\mu}(-1)^{k} \frac{\sigma_{k}\left(\alpha_{1}, \ldots, \alpha_{\mu}\right) \sigma_{\mu-k}\left(\beta_{1}, \ldots, \beta_{\nu}\right)}{\left(\begin{array}{c}
\nu \\
\mu-k
\end{array}\right)} .
$$

Our method of proof consists of showing that the right-hand side of (2.16) is precisely the multivariate Taylor expansion of $F$ at the origin $(\underline{\alpha}=\mathbf{0})$, as clearly given by

$$
F(\underline{\alpha})=\sum_{k=0}^{\mu} \frac{1}{k !} \sum_{\substack{1 \leq j_{\ell} \leq \mu \\ \ell=1, \ldots, k \\ j \ell \text { distinct }}} \frac{\partial^{k} F}{\partial \alpha_{j_{1}} \partial \alpha_{j_{2}} \ldots \partial \alpha_{j_{k}}}(\mathbf{0}) \alpha_{j_{1}} \alpha_{j_{2}} \cdots \alpha_{j_{k}} .
$$

Next, observing that the order in which the components of $\underline{\alpha}$ appear in the definition (2.15) of $F$ can be permuted arbitrarily without changing $F(\underline{\alpha})$, we see, in (2.17), that

$$
\begin{aligned}
& \frac{\partial^{k} F}{\partial \alpha_{j_{1}} \partial \alpha_{j_{2}} \ldots \partial \alpha_{j_{k}}}(\mathbf{0}) \\
& \quad=(-1)^{k}(\nu-\mu+k) \cdots(\nu-\mu+1) \sum_{i_{1}=1}^{\nu} \beta_{i_{1}} \sum_{\substack{i_{2}=1 \\
i_{2} \neq i_{1}}}^{\nu} \beta_{i_{2}} \ldots \sum_{\substack{i_{\mu-k}=1 \\
i_{\mu-k} \neq i_{1}, \ldots, i_{\mu-k-1}}}^{\nu} \beta_{i_{\mu-k}} \\
& \quad=(-1)^{k} \frac{(\nu-\mu+k) !}{(\nu-\mu) !}(\mu-k) ! \sigma_{\mu-k}\left(\beta_{1}, \cdots, \beta_{\nu}\right)
\end{aligned}
$$

which, together with (2.17), easily yields the desired result. 
If we now choose, in the identity (2.14), $\mu=\rho-1, \nu=n-1, p=\phi_{i+j, r, j}$ and $q=\psi_{2 i+2 j+r}$, we find that the expression (2.10) can be written in the explicit form

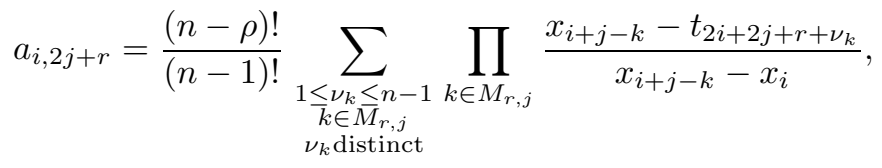

where the integer sets $M_{r, j}=M_{\rho, \lambda, r, j}$ are defined by

$$
M_{r, j}:=\left\{-\left\lfloor\frac{\lambda+r}{2}\right\rfloor,-\left\lfloor\frac{\lambda+r}{2}\right\rfloor+1, \ldots,-\left\lfloor\frac{\lambda+r}{2}\right\rfloor+\rho-1\right\} \backslash\{j\} .
$$

We have thus established the following result.

Theorem 2.2. For a knot sequence $\mathbf{t}$ satisfying (1.15), and with $n, \rho, \lambda$ denoting integers such that $n \geq 3,1 \leq \rho \leq n, \lambda \in \mathbb{Z}$, there exists a unique sequence $\left\{u_{\rho, \lambda, i}: i \in \mathbb{Z}\right\} \subset \mathcal{S}_{n, t}$, as given explicitly by (2.9), (2.18), such that (2.1) and (2.3) are satisfied.

Remark. It is clear from Theorem 2.2 that $u_{\rho, \lambda, i}$ is the minimally supported spline function in $\mathcal{S}_{n, \mathbf{t}}$ for which (2.3) holds; in this sense, we call the approximation operator $U_{\rho, \lambda}$ optimally local. (Note that this should not be confused with the optimally local interpolation operators $L_{n, \nu}$ introduced in (1.12).)

2.2. Applications of the quasi-interpolation and local interpolation blending formula. Next we explicitly construct, with the knot sequence $\mathbf{t}$ satisfying (1.15), and for positive integers $n, \rho$ and $\mu$ satisfying $n \geq 3,1 \leq \rho \leq n$, $1 \leq \mu \leq \max \left\{n+\rho-3,\left\lfloor\frac{3 n}{2}\right\rfloor+\rho-5\right\}$, a sequence of spline functions

$$
v_{\rho, \mu, i}=v_{\rho, \mu, n, \mathbf{t}, i} \in \mathcal{S}_{n, \mathbf{t}}, \quad i \in \mathbb{Z},
$$

which possess the following properties:

$$
\begin{gathered}
v_{\rho, \mu, i}\left(x_{j}\right)=\delta_{i, j}, \quad i, j \in \mathbb{Z} ; \\
\operatorname{supp} v_{\rho, \mu, i} \subseteq \begin{cases}{\left[x_{i-\mu}, x_{i-\mu+\rho+1}\right]} & \text { for } n=3, \\
{\left[x_{i-\mu}, x_{i-\mu+\lfloor 3 n / 2\rfloor+\rho-4}\right]} & \text { for } n \geq 4 ;\end{cases}
\end{gathered}
$$

and such that the corresponding interpolation operator $V_{\rho, \mu}=V_{\rho, \mu, n, \mathbf{t}}$ defined by

$$
\left(V_{\rho, \mu} f\right)(x)=\sum_{i \in \mathbb{Z}} f\left(x_{i}\right) v_{\rho, \mu, i}(x)
$$

reproduces all polynomials of order $\rho$, namely

$$
V_{\rho, \mu} p=p, \quad p \in \pi_{\rho-1} .
$$

Consider first the quadratic case with $n=3$. Appealing to Theorem 2.2, as well as $(2.1)$, we see that

$$
\operatorname{supp} u_{\rho, 2 \mu, i} \subseteq\left[x_{i-\mu}, x_{i-\mu+\rho+1}\right], \quad n=3 .
$$


Also, introducing for fixed $j \in \mathbb{Z}$ the Lagrange fundamental polynomials $\eta_{j, i}=$ $\eta_{\rho, \mu, j, i} \in \pi_{\rho-1}, i=1,2, \ldots, \rho$, by

$$
\eta_{j, i}(x)=\prod_{\substack{r=1 \\ r \neq i}}^{\rho} \frac{x-x_{j+\mu-r}}{x_{j+\mu-i}-x_{j+\mu-r}}, \quad i=1,2, \ldots, \rho,
$$

we deduce from (2.3) and (2.4) that

$$
\begin{aligned}
\delta_{\mu, i}=\eta_{j, i}\left(x_{j}\right) & =\sum_{q=1}^{\rho} \eta_{j, i}\left(x_{j+\mu-q}\right) u_{\rho, 2 \mu, j+\mu-q}\left(x_{j}\right) \\
& =u_{\rho, 2 \mu, j+\mu-i}\left(x_{j}\right),
\end{aligned}
$$

which, together with (2.24), implies that

$$
u_{\rho, 2 \mu, i}\left(x_{j}\right)=\delta_{i, j}, \quad i, j \in \mathbb{Z}, \quad n=3 .
$$

Thus, if we define $v_{\rho, \mu, i}:=u_{\rho, 2 \mu, i}$ for $n=3$, it follows immediately from (2.24), (2.25), and (2.3) that $v_{\rho, \mu, i}$ indeed satisfies the desired properties (2.20), (2.21), and (2.22), (2.23). For $n \geq 4$, however, the interpolation property (2.25) does not hold; but for this general case, we can employ the Chui-Diamond blending formula $[6]$ as in the bottom line of the construction (2.26) below. The main result of this section is as follows.

Theorem 2.3. Suppose the knot sequence $\mathbf{t}$ satisfies (1.15), and let $n, \rho$ and $\mu$ denote positive integers such that $n \geq 3,1 \leq \rho \leq n, 1 \leq \mu \leq \max \left\{n+\rho-3,\left\lfloor\frac{3 n}{2}\right\rfloor+\right.$ $\rho-5\}$. Then the sequence $v_{\rho, \mu, i} \in \mathcal{S}_{n, \mathbf{t}}, i \in \mathbb{Z}$, defined by

$$
v_{\rho, \mu, i}:= \begin{cases}u_{\rho, 2 \mu, i}, & \text { for } n=3, \\ \ell_{n, \tau_{n, \mu}, i}+u_{\rho, 2 \sigma_{n, \mu}, i}-L_{n, \tau_{n, \mu}} u_{\rho, 2 \sigma_{n, \mu}, i} & \text { for } n \geq 4,\end{cases}
$$

with

$$
\tau_{n, \mu}:=\min \{\mu, n-3\}, \quad \sigma_{n, \mu}:=\mu-\tau_{n, \mu}+1,
$$

and where $\ell_{n, \nu, i}$ and $L_{n, \nu}$ for $1 \leq \nu \leq \max \{1, n-3\}$, and $u_{\rho, \lambda, i}$ for $\lambda \in \mathbb{Z}$ are defined as in (1.11), (1.12), (2.9) and (2.18), satisfies the properties (2.20) and (2.21), as well as (2.22) and (2.23).

Remark. Observe from (1.12) and (2.1) that, for $n \geq 4,(2.26)$ can be written in the more explicit form

$$
v_{\rho, \mu, i}:=\ell_{n, \tau_{n, \mu}, i}+u_{\rho, 2 \sigma_{n, \mu}, i}-\sum_{k=1}^{\lfloor n / 2\rfloor+\rho-1} u_{\rho, 2 \sigma_{n, \mu}, i}\left(x_{i-\sigma_{n, \mu}+k}\right) \ell_{n, \tau_{n, \mu}, i-\sigma_{n, \mu}+k} .
$$

Graphs showing the construction procedure of $v_{\rho, \mu, i}$ following (2.26), will be displayed in $\S 4$.

Proof of Theorem 2.3. By virtue of the discussion preceding Theorem 2.3, it will suffice to establish the theorem for $n \geq 4$. 
Noting first from (2.27) that $1 \leq \tau_{n, \mu} \leq n-3$, we deduce that $\ell_{n, \tau_{n, \mu}, i}$ and $L_{n, \tau_{n, \mu}}$, as appearing in (2.26), are indeed well defined. Next, recalling the support intervals (1.7) and (2.1), it follows from (2.28) that (2.21) holds. Also, applying (1.3) and (1.13) to (2.26), we can verify the interpolation property $(2.20)$.

Finally, to prove (2.22) and (2.23), we use (1.12) and (2.2) to write, for $p \in \pi_{\rho-1}$,

$$
V_{\rho, \mu} p=\left(L_{n, \tau_{n, \mu}}+U_{\rho, 2 \sigma_{n, \mu}}-L_{n, \tau_{n, \mu}} U_{\rho, 2 \sigma_{n, \mu}}\right) p
$$

and then apply (2.3).

Remarks. (a) If we extend the polynomial reproduction property (2.23) to include the value $\rho=0$ in the sense that

$$
V_{0, \mu} p \neq p \text { for } p(x)=1, \quad x \in \mathbb{R},
$$

we observe from (1.3) and (1.7) that the choices

$$
v_{0, \mu, i}:= \begin{cases}\ell_{3,1, i} & \text { for } n=3 \\ \ell_{n, \mu, i} & \text { for } n \geq 4\end{cases}
$$

can be included in Theorem 2.3, but where the support properties (2.21) must be replaced by

$$
\operatorname{supp} v_{0, \mu, i} \subseteq \begin{cases}{\left[t_{2 i-1}, x_{i+1}\right]} & \text { for } n=3 \\ {\left[x_{i-\mu}, x_{i-\mu+n-2}\right]} & \text { for } n \geq 4 .\end{cases}
$$

(b) In the paper [15] it is shown that, for the optimal order $(\rho=n)$ polynomial reproduction case, and with the definition (1.2) of $x$ changed to $x_{i}:=t_{(n-1) i}, i \in \mathbb{Z}$, the minimally supported spline approximant, constructed similarly to those in $\S 2.1$ above, automatically interpolates at $x$, a result which is of course consistent with the quadratic case $n=3$ of Theorem 2.3 above.

2.3. Optimally local interpolatory spline approximants. The question now arises whether the sequence $\left\{v_{\rho, \mu, i}: i \in \mathbb{Z}\right\}$, as constructed in Theorem 2.3, is optimally local in the sense that $\left\{v_{\rho, \mu, i}: i \in \mathbb{Z}\right\}$ is the unique sequence in $\mathcal{S}_{n, \mathbf{t}}$ satisfying (2.20), (2.21) and (2.22), (2.23). Indeed, for positive integers $n, \rho$ and $\mu$ satisfying $n \geq 3,1 \leq \rho \leq n$ and $1 \leq \mu \leq n+\rho-3$, which is easily seen to imply the conditions on $n, \rho$ and $\mu$ in Theorem 2.3, Dahmen, Goodman, and Micchelli, [11, Theorem 4.3.1] proved the existence of a unique sequence

$$
w_{\rho, \mu, i}=w_{\rho, \mu, n, \mathbf{t}, i} \in \mathcal{S}_{n, \mathbf{t}}, \quad i \in \mathbb{Z},
$$

which possesses the following three properties:

$$
\begin{gathered}
w_{\rho, \mu, i}\left(x_{j}\right)=\delta_{i, j}, \quad i, j \in \mathbb{Z} ; \\
\operatorname{supp} w_{\rho, \mu, i} \subseteq\left[x_{i-\mu}, x_{i-\mu+n+\rho-2}\right] ;
\end{gathered}
$$

and that the corresponding interpolation operator $W_{\rho, \mu}=W_{\rho, \mu, n, t}$ defined by

$$
\left(W_{\rho, \mu} f\right)(x)=\sum_{i \in \mathbb{Z}} f\left(x_{i}\right) w_{\rho, \mu, i}(x)
$$


reproduces all polynomials of order $\rho$, namely,

$$
W_{\rho, \mu} p=p, \quad p \in \pi_{\rho-1} .
$$

Note in particular that the uniqueness of $w_{\rho, \mu, i}$, together with its support property (2.30), imply that $w_{\rho, \mu, i}$ has minimum support. The procedure suggested in [11] for the construction of $w_{\rho, \mu, i}$ is to solve a system of linear equations of dimension $(n+2 \rho-3) \times(n+2 \rho-3)$.

Now recall the result of Theorem 2.3, from which it is easily verified that

$$
\operatorname{supp} v_{\rho, \mu, i} \subseteq\left[x_{i-\mu}, x_{i-\mu+n+\rho-2}\right], \quad 3 \leq n \leq 5,
$$

whereas

$$
\operatorname{supp} v_{\rho, \mu, i} \subseteq\left[x_{i-\mu}, x_{i-\mu+\lfloor 3 n / 2\rfloor+\rho-4}\right] \nsubseteq\left[x_{i-\mu+n+\rho-2}\right], \quad n \geq 6 .
$$

Hence, from the uniqueness part of the above mentioned Dahmen, Goodman, and Micchelli result, we deduce that

$$
v_{\rho, \mu, i}=w_{\rho, \mu, i}, \quad 3 \leq n \leq 5,
$$

whereas, for $n \geq 6$, the sequence $\left\{v_{\rho, \mu, i}, i \in \mathbb{Z}\right\}$ lacks the minimum support property (2.30) of $\left\{w_{\rho, \mu, i}: i \in \mathbb{Z}\right\}$, in the sense that the index difference between the two endpoints of the interval in (2.21) exceeds that of the interval in (2.30) by $\lfloor(n-4) / 2\rfloor$.

We can therefore conclude that, for $3 \leq n \leq 5$, our formulas (2.26) explicitly solve the Dahmen, Goodman, and Micchelli $(n+2 \rho-3) \times(n+2 \rho-3)$ linear system for the $B$-spline coefficients of $w_{\rho, \mu, i}$. For example, in the case $n=5, \rho=5$, $1 \leq \mu \leq 7$, the original $12 \times 12$ linear system is solved by our construction (2.28), in which all the quantities are explicitly given by the formulas (2.9), (2.18), as well as (1.11), in which the quotient of two second-order determinants needs to be evaluated. For $n \geq 6$, our construction for $v_{\rho, \mu, i}$ remains explicit, at the price of exceeding, in terms of index difference, the minimum support property of $w_{\rho, \mu, i}$ by the quantity $\lfloor(n-4) / 2\rfloor$.

\section{Construction OF SPLINE WAVELETS ON NONUNiform KNOtS}

Let $m \geq 2$ be any positive integer, and $\mathbf{t}, \mathbf{x}$ the two bi-infinite sequences defined by (1.1) and (1.2), respectively. We will be interested in the (closed) spline subspaces

$$
V_{\mathbf{x}, 0}^{m}:=\operatorname{clos}_{L^{2}}\left(\mathcal{S}_{m, \mathbf{x}}\right) \quad \text { and } \quad V_{\mathbf{t}, 1}^{m}:=\cos _{L^{2}}\left(\mathcal{S}_{m, \mathbf{t}}\right)
$$

of $L^{2}:=L^{2}(\mathbb{R})$. Note that $V_{\mathbf{x}, 0}^{m}$ is a subspace of $V_{\mathbf{t}, 1}^{m}$, and we will be concerned with the orthogonal complement $W_{\mathbf{t}, \mathbf{x}, 0}^{m}$ in $V_{\mathbf{t}, 1}^{m}$ of $V_{\mathbf{x}, 0}^{m}$. While the $B$-splines $N_{m, \mathbf{x}, i}$ and $N_{m, \mathbf{t}, i}, i \in \mathbb{Z}$, are minimally supported functions that generate $V_{\mathbf{x}, 0}^{m}$ and $V_{\mathbf{t}, 1}^{m}$, respectively (by taking all $\ell^{2}$-linear combinations), the minimally supported functions

$$
\psi_{m, i}=\psi_{m, \mathbf{t}, i} \in W_{\mathbf{t}, \mathbf{x}, 0}^{m} \subset V_{\mathbf{t}, 1}^{m}, \quad i \in \mathbb{Z},
$$


that generate all of $W_{\mathbf{t}, \mathbf{x}, 0}^{m}$ (again by taking $\ell^{2}$-linear combinations) are called $B$ spline-wavelets (or simply $B$-wavelets) of order $m$ on $\mathbf{t}$.

To identify the space $W_{\mathbf{t}, \mathbf{x}, 0}^{m}$, we follow $[8,10]$ (for the case of uniform knot distributions; see also [5, Theorem 6.2]) and [2] (for nonuniform simple knot distributions), by introducing the space

$$
V_{\mathbf{t}, 1}^{2 m, 0}:=\left\{f \in V_{\mathbf{t}, 1}^{2 m}:\left.f\right|_{\mathbf{x}}=0\right\}
$$

and observe that although the knot sequence $\mathbf{t}$ in (1.1) allows multiplicities, the same proof as in the case of uniform knot distribution gives the isomorphism between $V_{\mathbf{t}, 1}^{2 m, 0}$ and $W_{\mathbf{t}, \mathbf{x}, 0}^{m}$ by using the $m$-fold differentiation $D^{m}$. Hence, by identifying the minimally supported functions

$$
\Psi_{2 m, i}=\Psi_{2 m, \mathbf{t}, i} \in V_{\mathbf{t}, 1}^{2 m, 0}, \quad i \in \mathbb{Z},
$$

the $m$ th-order $B$-wavelets in (3.2) are given by

$$
\psi_{m, i}=D^{m} \Psi_{2 m, i}, \quad i \in \mathbb{Z}
$$

If we require $x_{i}$ to be the left-hand endpoint of supp $\Psi_{2 m, i}$, then it is not difficult to see that in order to satisfy the interpolation condition $\Psi_{2 m}\left(x_{i+j}\right)=0, j=$ $1,2, \ldots$, the (nontrivial) $\Psi_{2 m, i}$ with minimum support is a linear combination of the $B$-splines

$$
N_{2 m, 2 i}, \quad N_{2 m, 2 i+1}, \ldots, \quad N_{2 m, 2 i+2 m-2} .
$$

Hence, in general, we have

$$
\operatorname{supp} \Psi_{2 m, i}=\left[x_{i}, x_{i+2 m-1}\right] .
$$

On the other hand, by applying the optimally local operator $L_{2 m}$ in (1.14) (with $n=2 m$ ), it is clear that

$$
V_{\mathbf{t}, 1}^{2 m, 0}=\left\{\phi-L_{2 m} \phi: \phi \in V_{\mathbf{t}, 1}^{2 m}\right\}
$$

So, to construct $\Psi_{2 m, i}$, we must find $\phi \in V_{\mathbf{t}, 1}^{2 m}$ such that $\phi-L_{2 m} \phi$ vanishes outside $\left[x_{i}, x_{i+2 m-1}\right]$.

For this purpose, we introduce the knot sequence $\tilde{\mathbf{t}}=\tilde{\mathbf{t}}(i):=\mathbf{t} \backslash\left\{x_{i+m-1}, x_{i+m}\right\}$ and set

$$
\tilde{\mathbf{t}}: \cdots \leq \tilde{t}_{-1} \leq \tilde{t}_{0} \leq \cdots
$$

with

$$
\tilde{t}_{j}= \begin{cases}t_{j} & \text { for } \quad j \leq 2 i+2 m-3 \\ t_{2 i+2 m-1} & \text { for } \quad j=2 i+2 m-2 \\ t_{j+2} & \text { for } \quad j \geq 2 i+2 m-1 .\end{cases}
$$


As in (1.1)-(1.2), we also need the sequence of sample points

$$
\left\{\begin{array}{l}
\tilde{\mathbf{x}}=\tilde{\mathbf{x}}(i): \cdots<\tilde{x}_{-1}<\tilde{x}_{0}<\cdots, \\
\text { where } \quad \tilde{x}_{j}:=\tilde{t}_{2 j}, \quad j \in \mathbb{Z}
\end{array}\right.
$$

Hence, it follows from (3.8b) and (3.9a) that

$$
\tilde{x}_{j}=\left\{\begin{array}{lll}
x_{j} & \text { for } & j \leq i+m-2, \\
t_{2 i+m-1} & \text { for } & j=i+m-1, \\
x_{j+1} & \text { for } & j \geq i+m .
\end{array}\right.
$$

Now, instead of using the sequences $\mathbf{t}$ and $\mathbf{x}$ to determine the optimally local fundamental functions in (1.3), we consider the sequences $\tilde{\mathbf{t}}$ and $\tilde{\mathbf{x}}$, yielding

$$
\phi_{2 m, i}:=\ell_{2 m, i_{2 m}, \tilde{\mathbf{t}}, \tilde{\mathbf{x}}, i+m-1}=\ell_{2 m, m-1, \tilde{\mathbf{t}}, \tilde{\mathbf{x}}, i+m-1} .
$$

By referring to $(3.9 \mathrm{~b})$, it is then easy to verify from (1.3) and (1.8) the following properties of $\phi_{2 m, i}$ :

$$
\begin{aligned}
\operatorname{supp} \phi_{2 m, i} & =\left[\tilde{x}_{i}, \tilde{x}_{i+2 m-2}\right]=\left[x_{i}, x_{i+2 m-1}\right], \\
\phi_{2 m, i}\left(x_{j}\right) & =0 \text { for } j \leq i+m-2 \text { or } j \geq i+m+1 .
\end{aligned}
$$

So, by (3.11b) and using the optimally local operator $L_{2 m}$ in (1.14), which was determined by $\ell_{2 m, i}=\ell_{2 m, m-1, \mathbf{t}, \mathbf{x}, i}$ in (1.5), we have

$$
\left(L_{2 m} \phi_{2 m, i}\right)(x)=\phi_{2 m, i}\left(x_{i+m-1}\right) \ell_{2 m, i+m-1}(x)+\phi_{2 m, i}\left(x_{i+m}\right) \ell_{2 m, i+m}(x) .
$$

It now follows from (1.8) that

$$
\operatorname{supp}\left(L_{2 m} \phi_{2 m, i}\right)=\left[x_{i}, x_{i+2 m-1}\right]
$$

which agrees with the support of $\phi_{2 m, i}$ in (3.11a). Hence, in view of (3.6) and (3.7), we may set

$$
\Psi_{2 m, i}(x)=\phi_{2 m, i}(x)-\left(L_{2 m} \phi_{2 m, i}\right)(x) .
$$

This establishes the following.

Theorem 3.1. Let $\mathbf{t}$ and $\mathbf{x}$ be the knot sequences given by (1.1) and (1.2), respectively, and $m$ any positive integer. Then the $B$-wavelets that generate $W_{\mathbf{t}, \mathbf{x}, 0}^{m}$ are given by $\psi_{m, i}, i \in \mathbb{Z}$, in (3.5), where $\Psi_{2 m, i}$ are described in (3.12), with $\phi_{2 m, i}$ given by (3.10). 


\section{Graphs of interpolatory approximants AND WAVElEts}

We first demonstrate the construction procedure of the interpolatory approximants $v_{\rho, \mu, i}$ by using the formula in (2.26), with $n=5$. In Figure $1 \mathrm{a}-\mathrm{c}$, we display the graphs of $\ell_{5,2,10}, u_{3,4,10}$ and the interpolatory approximant $v_{3,3,10}$, with knot

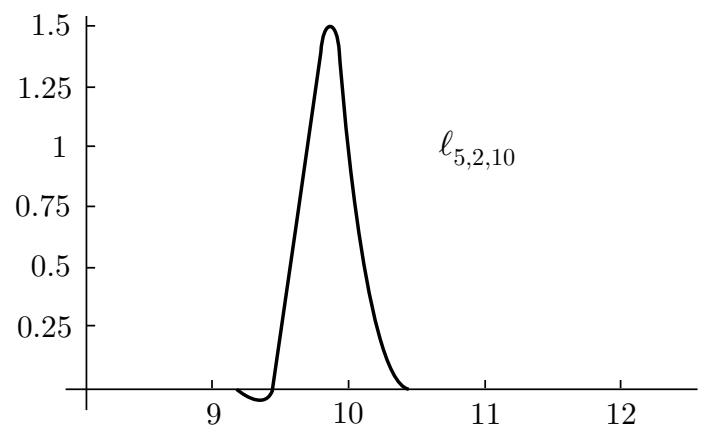

(a)

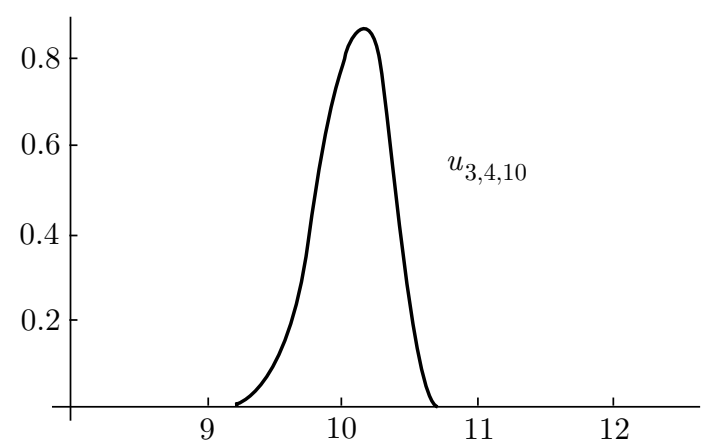

(b)

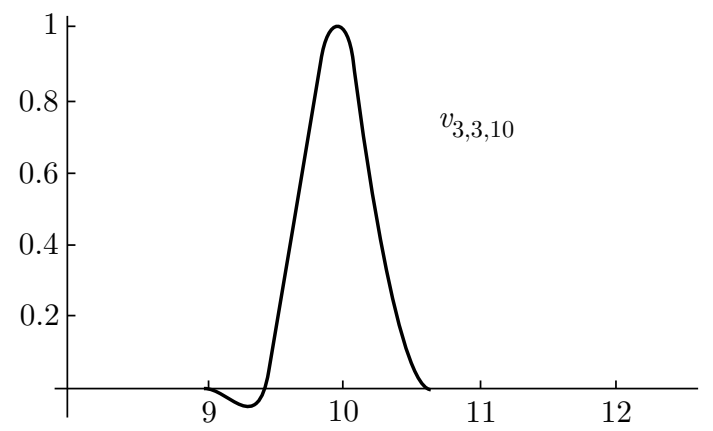

(c)

Figure 1. (a) $\ell_{5,2,10}$ (b) $u_{3,4,10}$ (c) $v_{3,3,10}$ 


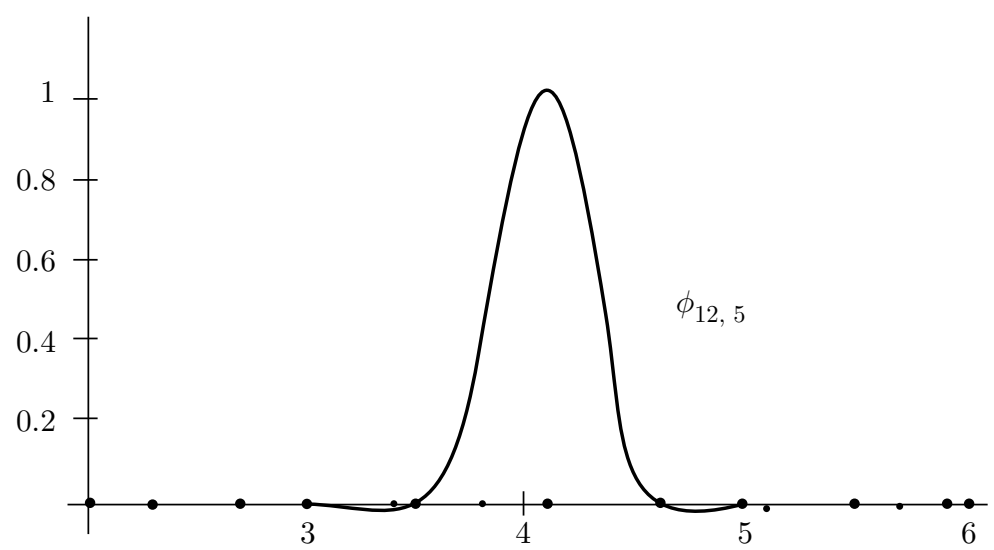

(a)

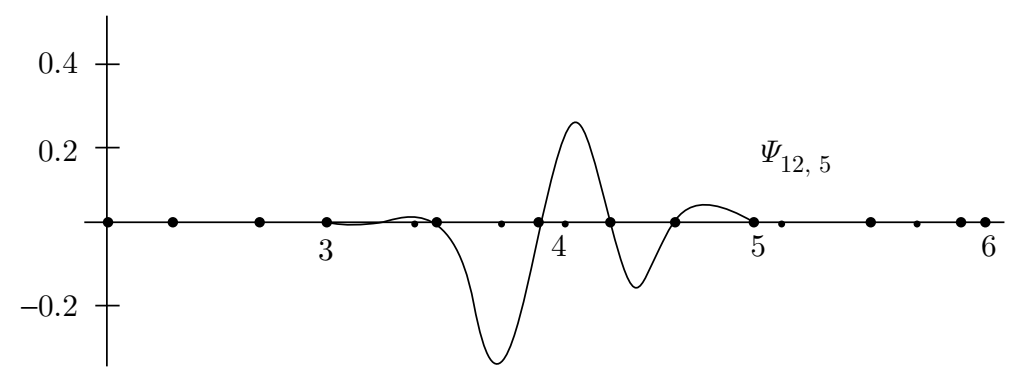

(b)

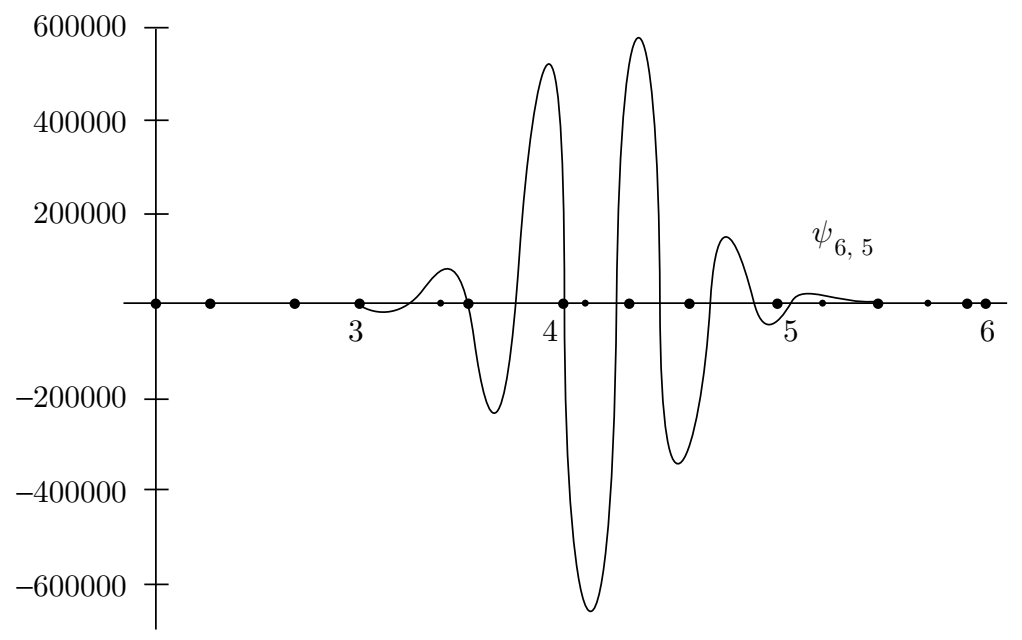

(c)

Figure 2. (a) $\phi_{12,5}$ (b) $\Psi_{12,5}$ (c) $\psi_{6,5}$ 
and node sequences

$$
\begin{aligned}
\mathbf{t}: \cdots & <4.8<5<5.2<5.3<5.5<6.3<6.5<7<7.6<7.8<7.9<7.95<8 \\
& <8.07<8.1<8.8<9<9.1<9.4<9.6<10<10.2<10.6<10.65<10.7 \\
& <10.9<11<11.5<12<12.07<12.1<\cdots, \\
\mathbf{x}: \cdots & <4.8<5.2<5.5<6.5<7.6<7.9<8<8.1<9<9.4<10<10.6<10.7 \\
& <11<12<12.1<\cdots,
\end{aligned}
$$

respectively, and with $t_{0}=x_{0}=4.8$. Observe that

$$
\operatorname{supp} \ell_{5,2,10}=[9,10.6], \quad \operatorname{supp} u_{3,4,10}=[9,11], \quad \operatorname{supp} v_{3,3,10}[8.1,11]
$$

Also observe that the indices of $\ell_{n, \tau_{n, \mu}, i}, u_{\rho, 2 \sigma_{n, \mu}, i}$, and $v_{\rho, \mu, i}$ come from the choice of

$$
n=5, \rho=3, \mu=3, i=10 \text {, }
$$

so that

$$
\tau_{n, \mu}=\min \{3,2\}=2 \quad \text { and } \quad \sigma_{n, \mu}=3-2+1=2 .
$$

To demonstrate the construction of the spline wavelets $\psi_{m, i}$ with nonuniform knots, we follow the procedure (3.12) and (3.5) with $\phi_{2 m, i}$ given by (3.10). The graphs of $\phi_{2 m, i}, \Psi_{2 m, i}$, and $\psi_{m, i}$, with $m=6, i=5$, and knot and node sequences

$$
\begin{aligned}
\mathbf{t}: \cdots & <0<0.2<0.4<0.5<0.6<0.9<1<1.2<1.3<1.5 \\
& <2=2<2.3<2.7=2.7 \\
& <3=3<3.4<3.5<3.8<4<4.1<4.3<4.6=4.6<5=5<5.1<5.5 \\
& <5.7<5.9<6=6<6.2<7=7<7.5<8=8<8.2<9 \cdots \\
\mathbf{x}: \cdots & <0<0.4<0.6<1<1.3<2<2.3<2.7<3<3.5<4<4.3<4.6 \\
& <5<5.5<5.9<6<7<7.5<8<9 \cdots
\end{aligned}
$$

respectively, and $t_{0}=x_{0}=0$, are shown in Figure $2 \mathrm{a}-\mathrm{c}$. Observe that with $i=5$, we have

$$
\operatorname{supp} \phi_{12,5}=\operatorname{supp} \Psi_{12,5}=\operatorname{supp} \psi_{6,5}=\left[x_{5}, x_{16}\right]=[2,6]
$$

\section{ACKNOWLEDGMENT}

We would like to thank Christophe Birkeland for letting us use the program from his report to NTH, University of Trondheim, Norway, Spring 1993, to produce the graphs in this paper.

\section{REFERENCES}

1. C. de Boor, A practical guide to splines, Appl. Math. Sci. \#27, Springer-Verlag, New York, 1978. MR 80a:65027

2. M. Buhmann and C. A. Micchelli, Spline pre-wavelets for non-uniform knots, Numer. Math. 61 (1992), 455-474. MR 93g:41021 
3. G. Chen, C. K. Chui, and M. J. Lai, Construction of real-time spline quasi-interpolation schemes, Approx. Theory Appl. 4 (1988), 61-75. MR 90d:41015

4. C. K. Chui, Construction and applications of interpolation formulas, Multivariate Approximation and Interpolation (W. Haussmann and K. Jetter, eds.), Internat. Ser. Numer. Math., vol. 94, Birkhäuser Verlag, Basel, 1990, pp. 11-23. MR 92c:41002

5. __ An introduction to wavelets, Academic Press, Boston, 1992. MR 93f: 42055

6. C. K. Chui and H. Diamond, A general framework for local interpolation, Numer. Math. 58 (1991), 569-581. MR 92b:41005

7. C. K. Chui and E. Quak, Wavelets on a bounded interval, Numerical Methods in Approximation Theory, vol. 9 (D. Braess and L.L. Schumaker, eds.), Internat. Ser. Numer. Math., vol. 105, Birkhäuser Verlag, Basel, 1992, pp. 53-75. MR 95b:42027

8. C. K. Chui and J. Z. Wang, A cardinal spline approach to wavelets, Proc. Amer. Math. Soc. 113 (1991), 785-793. MR 92b:41019

9. - On compactly supported spline wavelets and a duality principle, Trans. Amer. Math. Soc. 330 (1992), 903-915. MR 92f: 41020

10. 94f: 42041

11. W. Dahmen, T. N. T. Goodman, and C. A. Micchelli, Compactly supported fundamental functions for spline interpolation, Numer. Math. 52 (1988), 639-664. MR 89i:65011

12. Local spline interpolation schemes in one and several variables, Approximation and Optimization, Lecture Notes in Math., vol. 1354, Springer, Berlin, 1989, pp. 11-24. MR 90b: 41017

13. I. Daubechies, Ten lectures on wavelets, CBMS-NSF Regional Conference Series in Applied Math. \#61, SIAM, Philadelphia, PA, 1992. MR 93e:42045

14. J. M. De Villiers, A convergence result in nodal spline interpolation, J. Approx. Theory $\mathbf{7 4}$ (1993), 266-279. MR 94i:41013

15. J. M. De Villiers and C. H. Rohwer, Optimal local spline interpolants, J. Comput. Appl. Math. 18 (1987), 107-119. MR 88i:41017

16. - A nodal spline generalization of the Lagrange interpolant, Progress in Approximation Theory (P. Nevai and A. Pinkus, eds.), Academic Press, San Diego, 1991, pp. 201-212. MR 92h:41004

17. _ Sharp bounds for the Lebesgue constant in quadratic nodal spline interpolation, Approximation and Computation (R.V.M. Zahar, ed.), Internat. Ser. Numer. Math. \#119, Birkhäuser Verlag, Basel, 1994, pp. 157-167.

18. G. H. Golub and C. F. Van Loan, Matrix computations, Johns Hopkins Univ. Press, Baltimore, MD, 1985. MR 85h:65063

19. T. N. T. Goodman, S. L. Lee, and W. S. Tang, Wavelets in wandering spaces, Trans. Amer. Math. Soc. 338 (1993), 639-654. MR 93j:42017

20. S. Karlin, Total positivity, Stanford Univ. Press, Stanford, CA, 1968. MR 37:5667

21. T. Lyche and K. Mørken, Spline-wavelets of minimum support, Numerical Methods in Approximation Theory, Vol. 9 (D. Braess and L.L. Schumaker, eds.), Internat. Ser. Numer. Math., Vol. 105, Birkhäuser Verlag, Basel, 1992, pp. 177-192. MR 95c:41026

22. L. L. Schumaker, Spline functions: basic theory, Wiley-Interscience, New York, 1981. MR 82j: 41001

Center for Approximation Theory, Department of Mathematics, Texas A\&M UniverSity, College Station, Texas 77843

E-mail address: cchui@tamu.edu

Department of Mathematics, University of Stellenbosch, 7600 Stellenbosch, SOUTH AFricA

E-mail address: jmdv@sunvax.sun.ac.za 